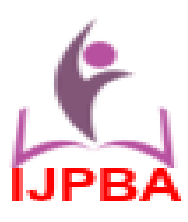

Contents lists available at www.ijpba.in

International Journal of Pharmaceutical and Biological Science Archive

PubMed (National Library of Medicine ID: 101738825)

Index Copernicus Value 2017: 71.80

Volume 7 Issue 2; March-April; 2019; Page No. 251-254

\title{
A REVIEW ON THE EFFECT OF FEBUXOSTAT IN CHRONIC KIDNEY PATIENTS, IT'S SIGNIFICANCE IN EGFR, URIC ACID AND ALBUMINURIA.
}

\author{
Jinsu Deena Jose ${ }^{1 *}$, Mathew George ${ }^{2}$, Lincy Joseph ${ }^{3}$ \\ ${ }^{1}$ Department of Pharmacy Practice, Pushpagiri College of Pharmacy, Thiruvalla-689107, Kerala, \\ India. \\ ${ }^{2}$ Department of Pharmacology, Pushpagiri College of Pharmacy, Thiruvalla-689107, Kerala, India. \\ ${ }^{3}$ Department of Pharmaceutical Chemistry, Pushpagiri College of Pharmacy, Thiruvalla-689107, \\ Kerala, India.
}

\section{ABSTRACT}

Chronic kidney disease is defined as the abnormality of the kidney structure or function for $\geq 3$ months and is associated with an irreversible reduction of the excretory and the endocrine functions of the kidney. An important risk factor for the development and progression of CKD is hyperuricemia. Hyperuricemia can occur as a result of the increased production or the reduced secretion of uric acid. Increased uric acid level is significantly associated with a greater decline in renal function and there is a higher risk of progression into kidney failure. Febuxostat is a nonpurine xanthine oxidase inhibitor for the treatment of hyperuricemia in patients with chronic kidney disease. It reduces serum uric acid concentrations by blocking the transformation of hypoxanthine to xanthine and xanthine to uric acid. Febuxostat is mainly metabolized in the liver and excreted through both urine and feces. Renal adjustment is also not required in CKD patients with mild to moderately reduced eGFR as it is metabolized mainly by glucuronidation and oxidation in the liver and well excreted by both urinary and fecal routes. Recent studies show that, in addition to lowering the uric acid level, febuxostat preserves the eGFR.

Keywords: Chronic kidney disease, hyperuricemia, febuxostat, eGFR

\section{INTRODUCTION}

Chronic kidney disease is defined as the abnormality of the kidney structure or function for $\geq 3$ months and is associated with an irreversible reduction of the excretory and the endocrine functions of the kidney ${ }^{(1)}$. The 5 stages of CKD are based on eGFR as recommended by professional guidelines. Radiolabeled procedures for the measurement of GFR are accurate however not practical, while the traditional methods require timed urine collection with its drawback of inaccuracy, cumbersomeness and inconvenient for the patients. Formula based calculation of eGFR has offered a very practical and easy approach for converting serum creatine value into GFR result taking into consideration patients age, sex, ethnicity and weight (depending on equation type). The most commonly used equations include Cockcroft and gault, modification of diet in renal disease $(\mathrm{MDRD})^{(2)}$.

An important risk factor for the development and progression of CKD is hyperuricemia. Hyperuricemia can occur as a result of the increased production or the reduced secretion of uric $\operatorname{acid}^{(3)}$. Increased uric acid level is significantly associated with a greater decline in renal function and there is a higher risk of progression into kidney failure. Normal uric acid levels are2.4-6.0 mg/dL (female) and 3.4$7.0 \mathrm{mg} / \mathrm{dL}$ (male). Purines plays an important role in blood uric acid levels. Purines are nitrogen containing compounds, which are made inside our cells (endogenous) or that come from outside of our body, i.e. from foods containing purines (exogenous). Purines breaks down into uric acid. Increased levels of uric acid 
from excess purines accumulates in our tissue and form crystals. This cause high uric acid level in blood. Causes of hyperuricemia can be primary (due to purines) and secondary (due to another disease or condition). Sometimes the body produces a lot of uric acid than it is able to excrete.

The measurement of urinary albumin is important because it is relatively standardized and this the single most important protein lost in the urine in most CKD. There is substantial evidence linking increased albuminuria to outcomes of CKD. The leakage of even small amount of albumin in urine is one of the earliest sign of a symptomatic kidney damage. Recent studies show that albuminuria is implicated in causal pathway of progression of kidney disease, this means that albuminuria can be a modifiable risk factor and that targeting and lowering of albuminuria will lead to renoprotection ${ }^{(4)}$.

Febuxostat is a nonpurine xanthine oxidase inhibitor for the treatment of hyperuricemia in patients with chronic kidney disease. It reduces serum uric acid concentrations by blocking the transformation of hypoxanthine to xanthine and xanthine to uric acid. Febuxostat is mainly metabolized in the liver and excreted through both urine and feces. Renal adjustment is also not required in CKD patients with mild to moderately reduced eGFR as it is metabolized mainly by glucuronidation and oxidation in the liver and well excreted by both urinary and fecal routes. Recent studies show that, in addition to lowering the uric acid level, febuxostat preserves the eGFR ${ }^{(5)}$.

\section{LITERATURE REVIEWS}

1. Mukri M N A et al (2018) conducted a prospective open label randomized study in chronic kidney disease stage 3 and 4 patients with diabetic nephropathy and asymptomatic hyperuricemia. Patients were randomized into febuxostat $40 \mathrm{mg}$ daily and no treatment group and were followed up for 6 months. Their usual take care for DM, hypertension and dyslipidemia were continued in the study. The eGFR in febuxostat group was stabilized at 6 months with no significant reduction whereas there was a significant reduction of the eGFR in no treatment group. Proteinuria level was unchanged in both groups ${ }^{(6)}$.

2. Whelton et al (2017) examined the longterm effect of urate lowering therapy with febuxostat on eGFR . 116 hyperuricemic gout subjects received daily doses of febuxostat $(40,80$ or $120 \mathrm{mg})$ for up to 5 years. Serum uric acid concentration and eGFR were assessed regularly. Maintenance or improvement in eGFR was inversely corelated with the quantitative reduction in serum uric acid from baseline ${ }^{(7)}$.

3. Sircar D et al (2015) conducted a single center double blind, randomized, parallel group, placebo-controlled study. Eligible participants were adults aged 18-65 years with chronic kidney disease stages 3 and 4, with asymptomatic hyperuricemia. The intervention group received febuxostat, $40 \mathrm{mg}$ once daily for six months whereas the placebo group received placebo, both the groups were followed up for six months. Mean eGFR in the febuxostat group showed a nonsignificant increase from $31.5 \pm 13.6$ (SD) to $34.7 \pm 18.1 \mathrm{ml} / \mathrm{min} / 1.73 \mathrm{~m}^{2}$ at 6 months. With placebo, mean eGFR decreased from a baseline of $32.6 \pm 11.6$ to $28.2 \pm 11.5$ $\mathrm{ml} / \mathrm{min} / 1.73 \mathrm{~m}^{2(8)}$.

4. Shigemasa Tani et al (2015) conducted a 6-month prospective study in which hypertensive hyperuricemic patients were randomized to either a febuxostat group $(n=30)$ or a control group $(n=30)$. The dose of febuxostat was adjusted to maintain the serum uric acid level at $<6.0 \mathrm{mg} / \mathrm{dL}$. In the febuxostat group the plasma renin activity (PRA), plasma aldosterone concentration (PAC) and serum uric acid level significantly decreased by $33 \%, 14 \%$ and $29 \%$ respectively. The eGFR significantly increased by $5.5 \%$, similar changes were not observed in control group ${ }^{(9)}$.

5. Ai Yu Yang et al (2018) conducted a retrospective study, which included patients with an estimated eGFR of $<30 \mathrm{ml} / \mathrm{min} / 1.73 \mathrm{~m}^{2}$ and hyperuricemia who received febuxostat. Changes were observed in the serum uric acid level, rate of achieving the target uric acid level $(<6.0 \mathrm{mg} / \mathrm{dL})$, changes in the eGFR, treatment dosage and adverse 
event.217 patients (65.9 \pm 15.1 yrs, 145 male and 72 females) with severe CKD and hyperuricemia were included. Febuxostat significantly lowered the serum uric acid level. The serum uric acid level was $<6 \mathrm{mg} / \mathrm{dL}$ in 126 patients. The change in eGFR was noted from $17.6 \pm 7.4$ at baseline and $17.8 \pm 8.9 \mathrm{ml} / \mathrm{min}$ after treatment. Adverse event was found in 5 patients, all adverse events improved after discontinuing febuxostat.

6. Yuki Tsuruta et al (2014) conducted a 1year cohort study of 73 hyperuricemic patients who had an eGFR below $45 \mathrm{ml} / \mathrm{min}$ and were being treated with urate lowering therapy. In 51 patients, treatment was changed from allopurinol to febuxostat and the other 22 patients were continued on allopurinol. The serum levels of uric acid level, creatinine and other biochemical parameter were measured at baseline and after 3,6,9 and12 months of treatment. The serum uric acid level significantly decreased from $6.1 \pm 1.0$ to $5.7 \pm 1.2 \mathrm{mg} / \mathrm{dL}$ in the febuxostat group and significantly increased from $6.2 \pm 1.1$ to $6.6 \pm 1.1$ $\mathrm{mg} / \mathrm{dL}$ in the allopurinol group. The eGFR decreased 27.3 to $25.7 \mathrm{ml} / \mathrm{min}$ in the febuxostat group and from 26.1 to $19.9 \mathrm{ml} / \mathrm{min}$ in the allopurinol group. Febuxostat reduced the serum uric acid levels and slowed the progression of renal disease in CKD cohort in comparison with allopurinol.

\section{CONCLUSION}

This review presents a detailed description about the effect of urate lowering therapy in chronic kidney patients. Hyperurecemia is a major contributor to the development or progression of chronic kidney disease. Urate lowering drugs such as allopurinol or febuxostat forestall chronic kidney disease progression and have a reno protective action by lowering the uric acid level, increasing eGFR, but no significant changes in proteinuria level.

\section{REFERENCES}

1. Levey AS, Inker LA. Definition and staging of chronic kidney disease in adults.http:// www.uptodate.com/contents/definition and-staging of CKD in adults.
2. Waad- Allah S, Mula-Abed, Khalid Al Rasadi, Dawood Al-Riyani.;Estimated glomerular filtration rate (eGFR) A serum creatinine based test for the detection of CKD and its impact on clinical practice; Oman Med.J.2012 March 27(2), 108-113.

3. Theodoros Eleftheriadis, Spyridon Golphino Poulos, Georgios Pissas and loannis Stefanidis; Asymptomatic hyperuricemia and chronic kidney disease; Narrative review of a treatment controversial; Journal of advanced research 2017 Sep:8(5) 555-560.

4. Hiddo J. Lambers Heerspink and Ron T.Gansevoort ;Albuminuria is an appropriate therapeutic target in patients with CKD: The pro view; Clinical Journal of American Society of Nephrology June 2015 10 (6) 1079-1088.

5. Tetsu Akimoto, Yoshiyuki Morishita, Chiharu Ito, Osamu Limura, Sadao Tsunematsu, Yuko Watanabe, Eiji Kusano, and Daisuke Nagata; Febuxostat for hyperuricemia in patients with advanced chronic kidney disease; Drug Target Insights.2014;8:39-43.

6. Mohd Noor Azreey Mukri, Wei-Yen Kong, Rizna Abdul Cader, Ruslinda Mustafar, Syahrul Sazliyana Shaharir, Shamsul Azhar Shah, Abdul Halim Abdul Gafor, Rozita Mohd, and Lydia Kamaruzaman; Role of febuxostat in retarding progression of diabetic kidney disease with asymptomatic hyperuricemia: A 6 months open-label, randomized controlled trial; EXCLI Journal 2018;17:563-575.

7. Whelton A, Macdonald PA, Zhaol, Hunt B, Gunawardhana L; Renal function in gout: long term treatment effect of febuxostat ; Journal of clinical rheumatology, January 2017 (1) 7-13.

8. Sircar D, Chatterjee S, Waikhom R, Golay V, Raychaudhury A, Pandey R; Efficay of febuxostat for slowing the GFR decline in patients with CKD and asymptomatic hyperuricemia A 6-month, double blind, randomized, placebo- controlled trial; American Journal of kidney disease 2015 December 66 (6) 945-950. 
9. Shigemasa Tani, Nagao K, Hirayama A; Effect of febuxostat, a Xanthine Oxidase inhibitor, on cardiovascular risk in hyperuricemia patient with hypertension a prospective, open label -pilot study; Clinical drug investigation 201535 (12) 823-831.

10. Ai Yu Yang; Febuxostat in patients with hyperuricemia and severe chronic kidney disease; African Journal of pharmacy and pharmacology 201812 (17) 193-201.

11. Yuki Tsuruta, Toshio Mochizuki, Mitsuvo Habashi, Takashi Taku; Switching from allopurinol to febuxostat for the treatment of hyperuricemia and renal function in patients with Chronic kidney patients; Journal of clinical rheumatology 2014 33(11) 1643-1648. 